\title{
Efficient harmonic microbunching in a 7th-order inverse-free-electron laser interaction
}

\author{
S. Ya. Tochitsky, ${ }^{1}$ O. B. Williams, ${ }^{2}$ P. Musumeci, ${ }^{2}$ C. Sung, ${ }^{1}$ D. J. Haberberger,${ }^{1}$ A. M. Cook, ${ }^{2}$ \\ J. B. Rosenzweig, ${ }^{2}$ and C. Joshi ${ }^{1}$ \\ ${ }^{1}$ Neptune Laboratory, Department of Electrical Engineering, UCLA, Los Angeles, California 90095, USA \\ ${ }^{2}$ Neptune Laboratory, Department of Physics and Astronomy, UCLA, Los Angeles, California 90095, USA
} (Received 19 August 2008; published 14 May 2009)

\begin{abstract}
We have shown that a seventh-order inverse-free-electron laser (IFEL) interaction, where the radiation frequency is the seventh harmonic of the fundamental resonant frequency, can microbunch a beam of relativistic electrons inside an undulator. Using coherent transition radiation (CTR) emitted by the bunched $12.3 \mathrm{MeV}$ beam as a diagnostic, strong microbunching of the beam is inferred from the observation of CTR at the first, second, and third harmonics of the seed $10 \mu \mathrm{m}$ radiation. Threedimensional IFEL simulations show that the observed harmonic ratios can be explained only if transverse spatial distribution of the steepened bunched beam is taken into account.
\end{abstract}

DOI: 10.1103/PhysRevSTAB.12.050703

PACS numbers: 41.60.Cr, 41.75.Jv, 41.75.Lx

There is a growing interest in developing new light sources in the $\mathrm{x}$-ray range with improved spatial and spectral coherence properties compared to the present-day synchrotron radiation sources. Many proposals and several ongoing national projects exist worldwide to build $\mathrm{x}$-ray or UV free-electron lasers (FELs) in which a high-brightness, multi-GeV electron beam has a resonant interaction with radiation in the presence of a periodic magnetic field of an undulator [1]. Because of the practical limit on the size and strength of undulator magnets, which all have periods in the $\mathrm{cm}$ range, the electron beam energy on the $\mathrm{GeV}$ scale represents one of the main constraints on the shortest reachable wavelength. To circumvent this limitation, a high-order FEL interaction has been proposed recently as a way to reduce the required beam energy [2]. In addition to FELs, inverse-free-electron laser (IFEL) acceleration [3], longitudinal current modulation of an electron beam (microbunching) [4], and creation of very short electron bursts (femtoslicing) [5] are other applications, which can potentially benefit from the high-order resonant beamradiation interaction in an undulator.

High-order FEL and IFEL interactions can be understood by looking closely at the resonant condition on the axis of a planar undulator:

$$
\lambda_{r}=\frac{\lambda_{u}}{2 n \gamma^{2}}\left(1+\frac{K_{u}^{2}}{2}\right)
$$

where $K_{u}=e B_{u} / m c k_{u}$ is the dimensionless undulator parameter, $\lambda_{r}$ is the radiation wavelength, $\gamma$ is the electron Lorentz factor, $\lambda_{u}$ the undulator wavelength, $n$ the harmonic number, $K_{u}$ the undulator wave number, and $B_{u}$ the undulator magnetic field [6]. A typical IFEL/FEL operates at the fundamental frequency $[n=1$ in Eq. (1)] providing the highest possible efficiency of the interaction. However, since the transverse velocity component of the electron motion in the undulator becomes relativistic at $K_{u} \geq 1$, resonance can also occur when the seed radiation frequency is a multiple of the fundamental resonant frequency [6]. In a collinear IFEL interaction in a planar undulator, the electron beam then may exchange energy with a seed radiation of wavelength $\lambda_{s}$ satisfying the condition $\lambda_{r}=$ $n \lambda_{s}$, where $n=3,5,7 \ldots$ (the high-order interactions). Unlike an atomic resonance, the high-order IFEL resonances for $n=7-11$ according to simulations [7] can be very efficient reaching an interaction strength of $50 \%$ of that at the $n=1$ case. If this efficiency is proved experimentally, a high-order coupling with $n \geq 3$ can add an important extra degree of freedom in designing systems when the energy of the beam is too low to tune the undulator at the $n=1$ resonance or the laser source is not available.

High-order IFEL interactions have been observed in proof-of-principle experiments when an undulator was seeded by $10 \mu \mathrm{m}$ [8] and $0.8 \mu \mathrm{m}$ [9] radiation pulses at a laser intensity of $2 \times 10^{14}$ and $2.6 \times 10^{12} \mathrm{~W} / \mathrm{cm}^{2}$, respectively. These studies have shown that high-order interactions do occur by observing energy modulation of the electrons whenever the beam energy, undulator parameters, and laser wavelength satisfy the resonant condition. On the other hand, microbunching of the beam inside the undulator has not been demonstrated and questions of the coupling efficiency and the applicability of the high-order interactions in future FEL and IFEL schemes remain open. Beam microbunching is induced by longitudinal motion of the particle in the periodic ponderomotive potential with a period of $\lambda_{\mathrm{mb}}=\lambda_{s}$ formed by the interaction of the radiation field with the undulator magnetic field. Characterization of the electron microbunching in the undulator yields directly a measure of the amplitude of the ponderomotive potential and so of the strength of the beam-radiation coupling [10,11]. A tightly microbunched beam has a steepened wave form and therefore a significant 
harmonic content at $\lambda_{\mathrm{mb}} / h$, where $h=1,2,3$, etc. is the harmonic number. Observation of harmonic components in the beam spectrum can then serve as a unique indicator of the strength and the efficiency of the beam-radiation coupling in the high-order $(n \geq 3)$ IFEL/FEL interactions.

This paper demonstrates for the first time efficient beamradiation coupling in the 7th order IFEL interaction. Here the undulator designed for a resonant wavelength of $74.2 \mu \mathrm{m}$ is seeded by a $\mathrm{CO}_{2}$ laser fulfilling the condition $10.6 \times 7=74.2 \mu \mathrm{m}[n=7$ in Eq. (1)]. Using coherent transition radiation (CTR) emitted by the bunched 12.3 MeV beam as a diagnostic, strong microbunching of the beam is inferred from the observation of CTR at the first, second, and third harmonics of the seed $10 \mu \mathrm{m}$ radiation. Comparison between the measured ratios of the second and third harmonic to the first harmonic in the CTR spectrum and the 3D IFEL simulations uncovered that an effective transverse beam size for each harmonic is different and should be taken into account. Observation of the steepened bunched beam at a laser intensity of $\sim 10^{9} \mathrm{~W} / \mathrm{cm}^{2}$ (3 orders of magnitude lower than in [9]) indicates that the high-order IFEL/FEL interactions have an efficiency comparable to that for the $n=1$ resonance and useful for practical applications.

The schematic of the experiment, carried out at the Neptune Laboratory at UCLA, is shown in Fig. 1. The Neptune rf photoinjector [12] provides a $\sim 12.3 \mathrm{MeV}$ electron beam with a charge up to $500 \mathrm{pC}$, a normalized emittance of $6 \mathrm{mmmrad}$, and a pulse length of $10 \mathrm{ps}$ (FWHM). The beam is injected into a $33 \mathrm{~cm}$ long planar permanent magnet undulator with a period of $3.3 \mathrm{~cm}$ and $K_{u}=1.8$. The beam is typically focused to a spot size of $\sim 350 \mu \mathrm{m}$ (rms) at the exit plane of the undulator where an insertable probe for a CTR screen is placed. The probe is also used for spatial alignment and temporal synchronization between the $\mathrm{CO}_{2}$ laser and the electron beam. The $10.6 \mu \mathrm{m} \mathrm{CO}$ laser beam is focused to a spot size of $650 \mu \mathrm{m}\left(1 / e^{2}\right)$ in the middle of the undulator using a $2.5 \mathrm{~m}$ focal length $\mathrm{NaCl}$ lens. This $\mathrm{F} / 100$ focusing provides an almost constant laser field intensity over the entire undulator length. A $100 \mathrm{ps}$ long $\mathrm{CO}_{2}$ laser pulse is used to seed the undulator with a peak power up to $34 \mathrm{MW}(2 \times$ $10^{9} \mathrm{~W} / \mathrm{cm}^{2}$ ). The beams are aligned in the middle and at

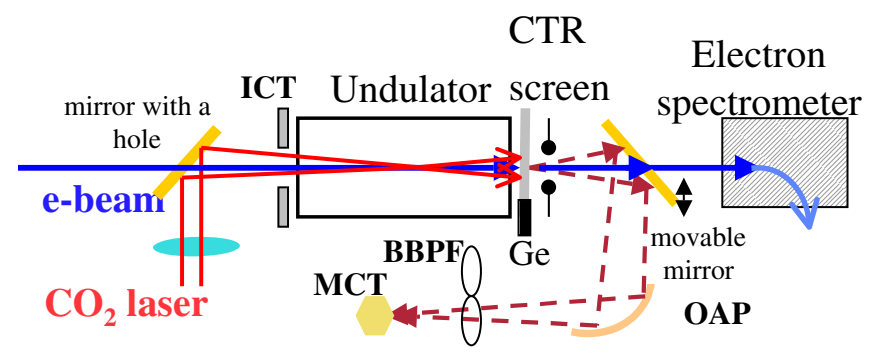

FIG. 1. (Color) A schematic of the experiment. ICT is an integrating current transformer. the exit of the undulator with a spatial accuracy of $100 \mu \mathrm{m}$ and an angular alignment of better than $1 \mathrm{mrad}$. After the undulator, the electron beam is sent to a high-resolution spectrometer to measure the energy spread.

The microbunching diagnostic consists of a CTR screen made of an $8 \mu \mathrm{m}$ thick $\mathrm{Al}$ foil, a movable mirror to transport the forward emitted CTR outside the vacuum chamber through a $\mathrm{NaCl}$ window, a focusing off-axis parabolic mirror, a set of broadband-pass filters $(\Delta \lambda \sim 1 \mu \mathrm{m})$ centered around the first $(10.6 \mu \mathrm{m})$, the second $(5.3 \mu \mathrm{m})$, and the third $(3.5 \mu \mathrm{m})$ harmonic wavelengths, and a liquid nitrogen cooled mercury cadmium telluride (MCT) detector. Even though the light-tight CTR screen is used to dump the pump laser beam, separation of the weak CTR component from the strong stray $10 \mu \mathrm{m}$ radiation is a critical issue. Two main measures are undertaken: first, the CTR foil screen is made larger than the undulator gap size and, second, a $6 \mathrm{~mm}$ diaphragm is placed in front of the pickup transport mirror limiting the field of view to the CTR radiation only. Together, these measures provide a rejection of the pump by a factor of $5 \times 10^{9}$ reducing the leakage background to $\sim 0.5 \mathrm{pJ}$. The signal-to-nose ratio of 25 at $10.6 \mu \mathrm{m}, 3(5 \mu \mathrm{m})$, and $1.6(3.5 \mu \mathrm{m})$ are reached in the experiment.

In order to inject the $10 \mathrm{ps}$ electron beam at the very maximum of the $100 \mathrm{ps}$ long $\mathrm{CO}_{2}$ laser pulse, the two must be synchronized on a picosecond time scale. Electronbeam-controlled transmission of $10 \mu \mathrm{m}$ radiation in $\mathrm{Ge}$ is utilized for the cross-correlation measurement to achieve temporal synchronization [13]. Here the $10 \mu \mathrm{m}$ pulse is sent through a 2-mm thick Ge plate mounted on the same insertable probe and the time dependence of the $10 \mu \mathrm{m}$ transmission is recorded. A typical result of the crosscorrelation measurement is presented in Fig. 2(a). If the electrons reach the Ge plate before the $\mathrm{CO}_{2}$ laser pulse, the $10 \mu \mathrm{m}$ pulse is fully attenuated by the free carriers generated by the electron beam. Then, as the electron beam is delayed, the electron beam and the $10 \mu \mathrm{m}$ pulse cross each other resulting in an increase of transmission. This is seen to be the case from 100 to $300 \mathrm{ps}$ in Fig. 2(a). By taking the derivative of curve fit of the data, an effective pulse length for the $\mathrm{CO}_{2}$ laser pulse is deduced to be $\sim 106 \mathrm{ps}$ (see the dashed curve). When the $\mathrm{Al}$ foil probe is inserted instead of the Ge plate and the beam energy is chosen to be $12.4 \mathrm{MeV}$, we observe time dependence of the CTR signal at $10 \mu \mathrm{m}$ shown in Fig. 2(b). The signal starts growing at $60 \mathrm{ps}$ and falls off to the background level at around 400 ps. This slightly larger time window for the CTR signal in comparison with the cross-correlation width in Fig. 2(a) can be attributed to a different laser intensity, $I$ scaling of microbunching: $\sqrt{I}$ and $I$, respectively [14]. However, the CTR output is clearly centered around $150-160$ ps where there is the maximum of the laser pulse in Fig. 2(a).

To prove the resonant character of interaction between the laser and the electron beam, we record the $10-\mu \mathrm{m} \mathrm{CTR}$ 

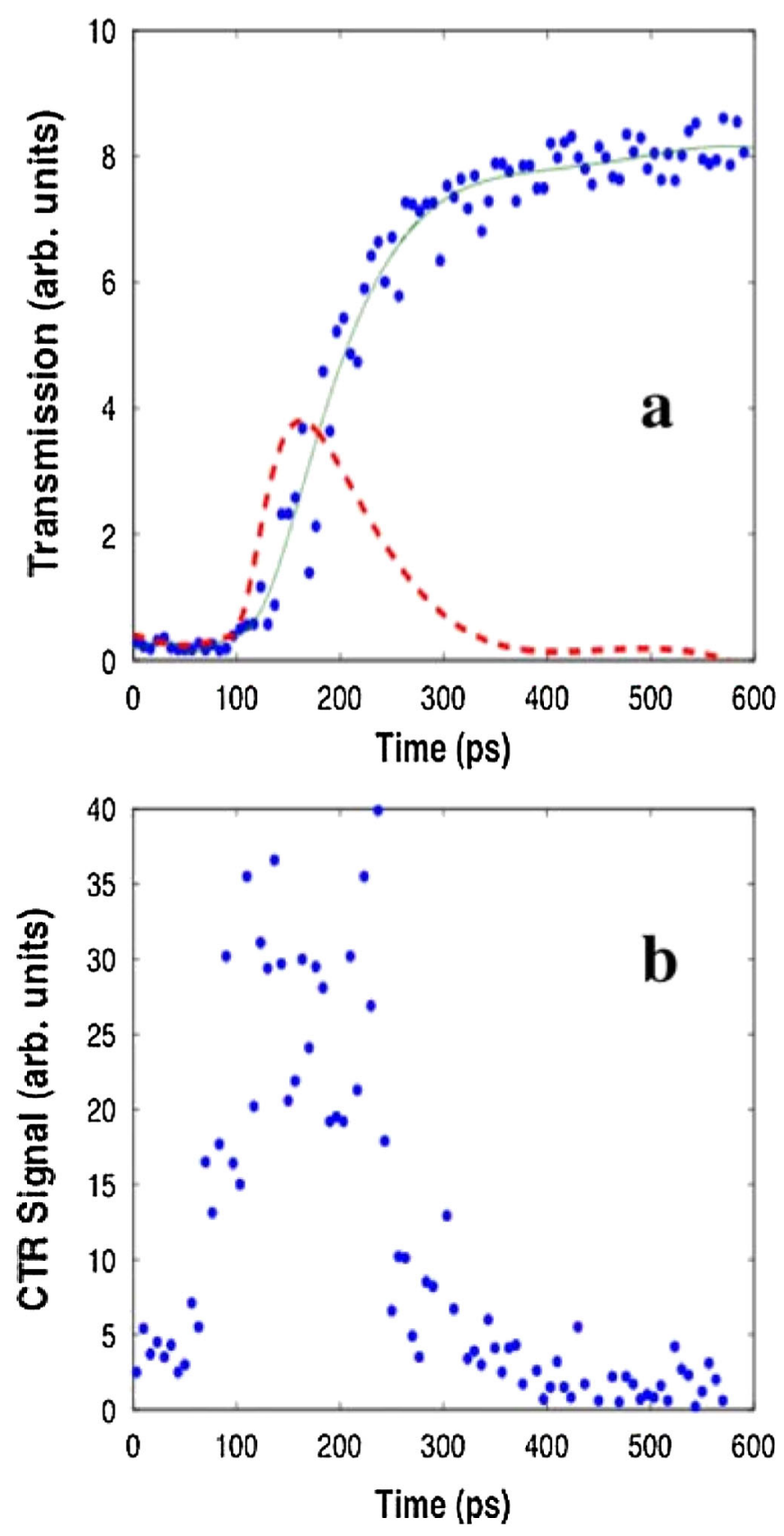

FIG. 2. (Color) The transmitted $10 \mu \mathrm{m}$ signal (a) and the CTR signal (b) as a function of the delay between the laser pulse and the electron bunch. The dashed curve is a derivative of curve fit of the data and represents the $\mathrm{CO}_{2}$ laser pulse profile.

signal for different energies of the electron beam and the results are summarized in Fig. 3. For these data we record the CTR signal, the laser power, and the electron beam charge for each interaction event, and each data point represents the mean value for 12 measurements with its standard deviation. The CTR output peaks around 12.5 MeV, which within the absolute energy measurement error agrees with the resonant energy for a 7th order IFEL interaction obtained from Eq. (1). The measured accep-

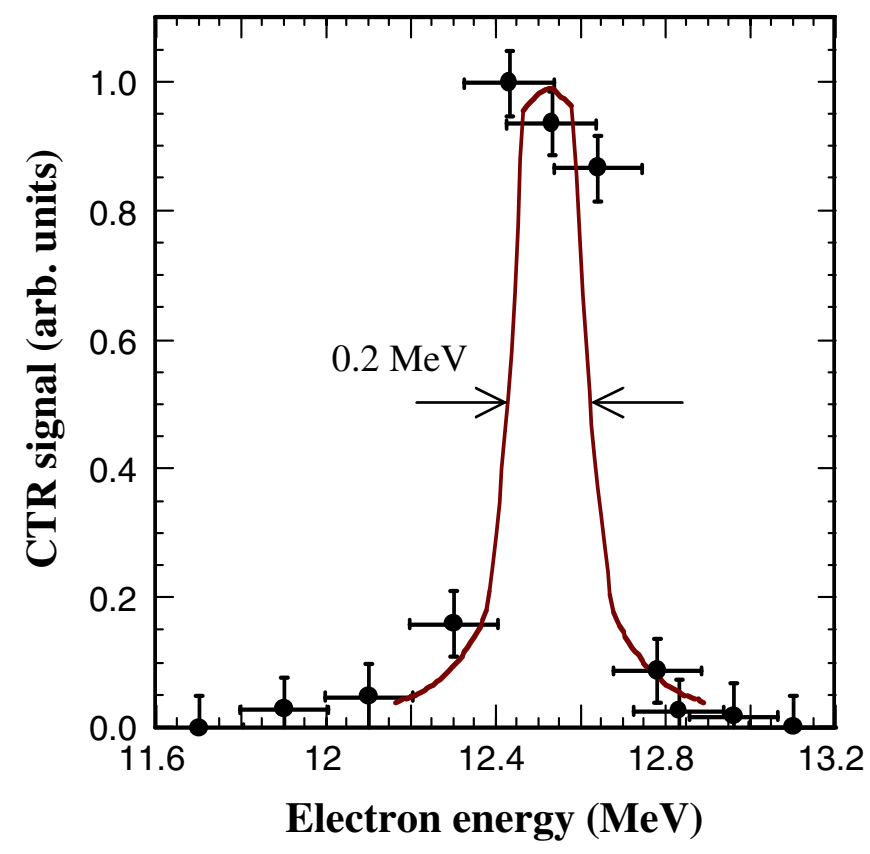

FIG. 3. (Color) Fundamental 10- $\mu \mathrm{m}$ CTR signal (circles) and the calculated normalized bunching factor (solid line) as a function of the electron beam energy.

tance energy width (FWHM) is $\sim 0.3 \mathrm{MeV}$ as opposed to the theoretical width of $0.2 \mathrm{MeV}$, deduced from the calculated bunching factor for different energies. The value of total $\mathrm{rms} \Delta E / E$ measured using the spectrometer (shown in Fig. 1) with and without the undulator is $\sim 90 \mathrm{keV}$ $(0.7 \%)$ and is presented as an uncertainty for each energy value in Fig. 3. Despite observation of a large CTR signal indicating a strong microbunching, due to this total energy spread we cannot measure energy modulation induced by the IFEL interaction. This can be explained by the fact that microbunching can occur whenever the local (slice) energy spread is smaller than the IFEL ponderomotive potential amplitude which in our case is estimated to be $50 \mathrm{keV}$. Ultimately, this local energy spread is limited by a thermal, uncorrelated energy spread which is less then $10 \mathrm{keV}$ in the case of a photoinjector. Thus, assuming that the electron beam has a correlated energy spread (the difference between the beam head and the beam tail energy), microbunching can occur for the whole beam when the central beam energy corresponds to the resonant energy for the 7th order IFEL interaction. Slight detuning of the central energy above (or below) the resonant value causes microbunching of a portion of the beam and can explain the broader measured width in Fig. 3.

Having established the optimal energy for the 7th order IFEL interaction, we undertake a study of the harmonic content of the bunched electron beam. The CTR process and its application to longitudinal microbunching measurements of a round, Gaussian electron beam has been extensively studied $[10,11]$. For a given harmonic, the CTR 
energy emitted in the forward direction is given by

$$
U_{h} \approx \frac{N^{2} e^{2} b_{h}^{2}}{4 \sqrt{\pi} \sigma_{z}}\left(\frac{\gamma}{h k_{s}}\right)^{4}\left(\frac{1}{\sigma_{x, y}}\right)^{4},
$$

where $N$ is the number of electrons in the bunch, $b_{h}=$ $1 / N \mid \sum_{i} e^{i h k_{s} z_{i} \mid}$ is the bunching factor for the $h$ th harmonic, $\sigma_{x, y}$ and $\sigma_{z}$ are the rms transverse size of a round beam $\left(\sigma_{x} \approx \sigma_{y}\right.$ ), and the longitudinal beam size, $k_{s}$ is the wave number of the radiation at the seed wavelength, and $z_{i}$ is a longitudinal position of particles.
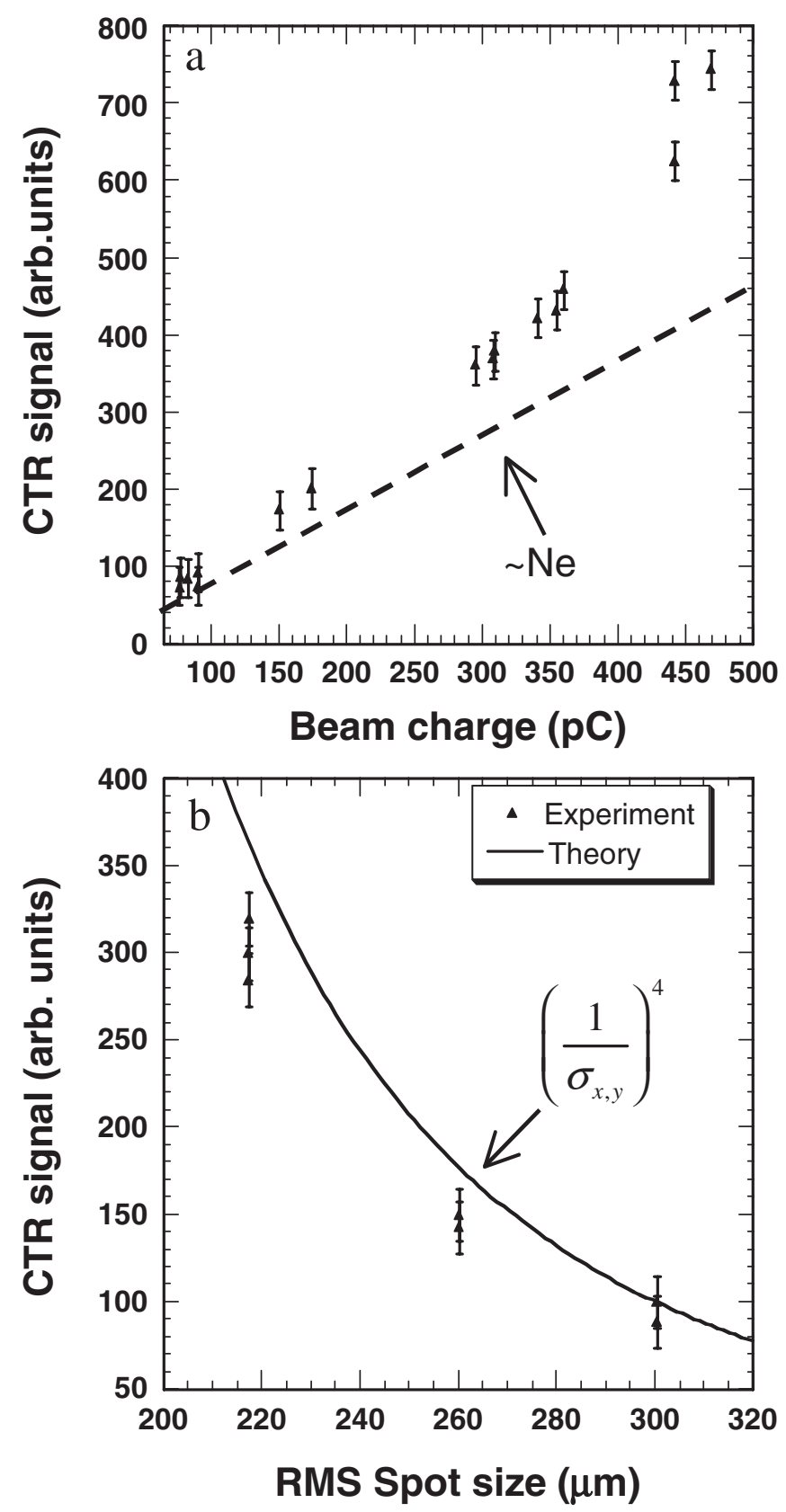

FIG. 4. Fundamental 10- $\mu \mathrm{m}$ CTR signal as a function of electron beam charge (a) and the transverse spot size $\sigma_{x, y}$ on the CTR screen at a fixed charge of $300 \mathrm{pC}$ (b).
The most prominent characteristic of the CTR is its $N^{2}$ scaling, which appears when the longitudinal electron beam size is comparable or smaller than the radiated wavelength. In Fig. 4(a) we present the CTR signal dependence upon the beam charge in the range of 80 to $450 \mathrm{pC}$. In the experiment we observe a clear nonlinear increase in the $10 \mu \mathrm{m}$ CTR signal by a factor of 10 . Note that this signal increase is observed only when both the beam and the undulator are present. However, the signal increase is weaker than one anticipated from $N^{2}$ scaling. This is mainly attributed to the worsening of the focusing ability for high charge beams experiencing the higher space charge force and slightly large emittance; therefore, the CTR level will be significantly reduced due to the increase in the beam size on the foil screen. Indeed, as shown in Fig. 4(b), for a fixed charge of $\sim 300 \mathrm{pC}$ we measure a factor of 3.2 decrease in the CTR level when the rms spot size $\sigma_{x, y}$ for the axisymmetric beam is increased from 220 to $300 \mu \mathrm{m}$. The $\left(1 / \sigma_{x, y}\right)^{4}$ scaling in Eq. (2) [see the solid curve in Fig. 4(b)] predicts a decrease by a factor of 3.5.

Figure 5 shows the CTR energy measurements for the first, $U_{1}\left(\lambda_{\mathrm{mb}}=\lambda_{s}\right)$, the second, $U_{2}\left(\lambda_{\mathrm{mb}}=\lambda_{s} / 2\right)$, and the third, $U_{3}\left(\lambda_{\mathrm{mb}}=\lambda_{s} / 3\right)$ harmonic components as a function of the laser power for the electron beam charge of $\sim 400$ pC. Note that for all data points here the background, which is proportional to the laser power, is subtracted. As seen in Fig. 5, at a low laser power of 3-19 MW only the $10 \mu \mathrm{m}$ CTR signal is detected. It is important that this seed laser power is comparable to that required for microbunching using the same undulator at the fundamental resonance [3]. Higher laser power causes bunching at

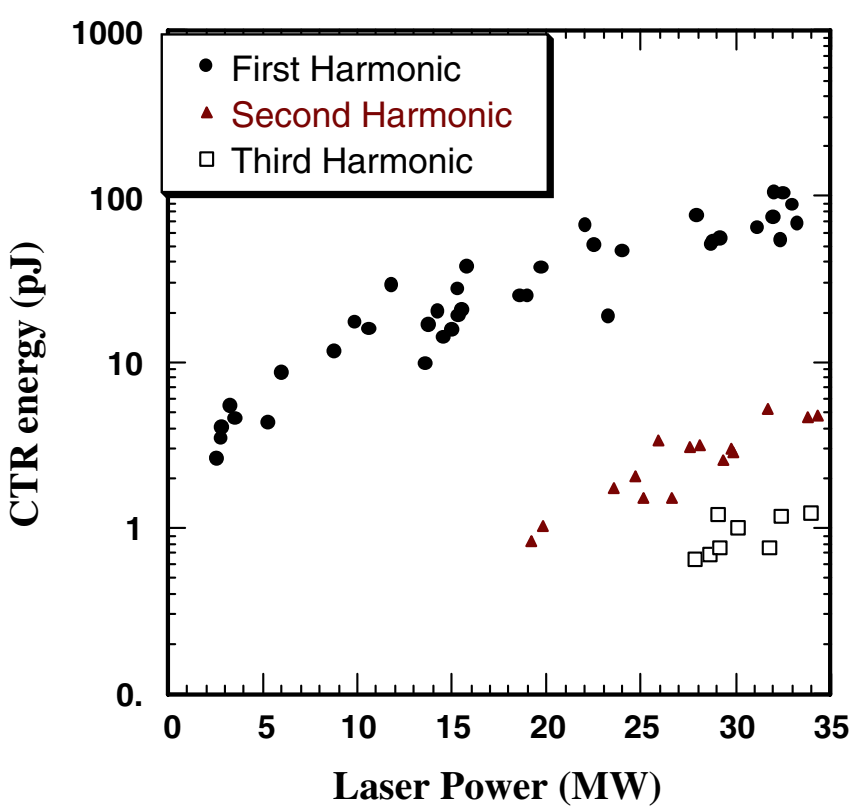

FIG. 5. (Color) CTR energy for the first (circles), second (triangles), and third harmonics (squares) as a function of the $\mathrm{CO}_{2}$ laser power. 
the first harmonic to occur earlier in the undulator giving rise to the second harmonic component for powers above 19 MW. A further increase in power speeds up the bunching process even more and for powers above $28 \mathrm{MW}$, the beam at the exit of the undulator has a third harmonic component. The measured ratios between the harmonic energy for the bunched beam are $U 1 / U 2 \approx 17 ; U 1 / U 3 \approx$ 70.

To compare these CTR measurements to a simulated longitudinal phase space of the beam, the 3D code TREDI is used to model the 7th order IFEL interaction [15]. Figure 6(a) plots the longitudinal phase space distribution of electrons at the exit of the undulator for a laser power of $34 \mathrm{MW}$. It is apparent that the electrons are bunched longitudinally on the $10.6 \mu \mathrm{m}$ scale and the wave form is steeper than a sinusoidal one. Furthermore, a color map in Fig. 6(a) indicates that there is a significant transverse variation in bunching and particles on axis (shown by dark blue color) are better bunched than the particles off axis (green color). The laser beam size in the experiment is approximately equal to the electron beam

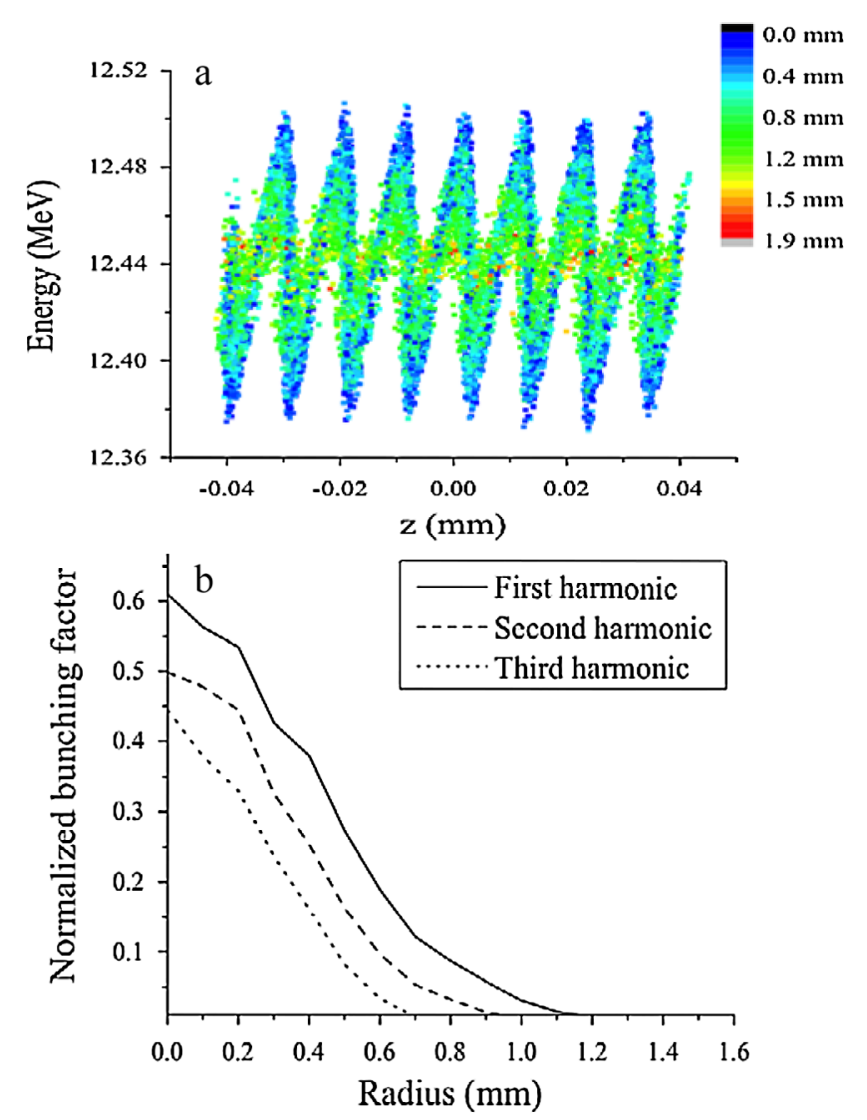

FIG. 6. (Color) TREDI simulations. Laser power $34 \mathrm{MW}, \sigma_{x, y}=$ $380 \mu \mathrm{m}$. (a) The longitudinal phase space distribution of electrons at the exit of the undulator and (b) normalized bunching coefficients for the first, second, and third harmonics of the bunched beam as a function of radius. The color code represents the radial distance of electrons from the beam axis. envelope size including the wiggling motion. In terms of the IFEL interaction, this represents a case where the offaxis particles see a smaller laser power and, therefore, are bunched more weakly. Simulations in Fig. 6(b) show radial distribution of the normalized bunching factor $b_{1}, b_{2}$, and $b_{3}$ for the first, the second, and the third harmonic components, respectively. The rms effective beam size in Fig. 6(b) is $380 \mu \mathrm{m}$ for the first, $335 \mu \mathrm{m}$ for the second, and $295 \mu \mathrm{m}$ for the third harmonics. This 3D effect significantly affects the measured harmonic ratios in the CTR spectrum. For the on-axis values of $b_{1}=0.6, b_{2}=0.5$, and $b_{3}=0.45$ and the above-mentioned $\sigma_{x, y}$, Eq. (2) predicts $U 1 / U 2=14$ and $U 1 / U 3=53$, which is in close agreement with the measurements in Fig. 5.

In conclusion, this experiment has shown microbunching of the electrons at the 7th order resonance from an IFEL. The electrons are efficiently bunched longitudinally inside a ten period long undulator producing the first, the second, and the third harmonics in a CTR spectrum. It is shown that, in the case of approximately equal sizes of the electron and the seed radiation beams, the IFEL interactions result in transverse variation of bunching that significantly affects the CTR harmonic content. Note that, in practically all seeded IFEL/FELs, 3D effects in beam matching may play a similar role and should be considered in CTR analysis. With the inclusion of the high-order IFEL/FEL interactions $(n \geq 3)$ on equal footage with the $n=1$ case, a significant flexibility can be gained in designing UV/x-ray FELs [2].

\section{ACKNOWLEDGMENTS}

The authors would like to thank W. Kimura (STI Optronics) for the undulator leasing and I. Pogorelsky (BNL) for contribution of the MCT detector. This work is supported by U.S. DOE Grants No. DE-FG0392ER40727 and No. DE-FG03-92ER40693.

[1] W. B. Colson, E. D. Johnson, M. J. Kelley, and H. A. Schwettman, Phys. Today, No. 1, 55, 35 (2002).

[2] B. W. J. McNeil, G. R. M. Robb, M. W. Poole, and N. R. Thompson, Phys. Rev. Lett. 96, 084801 (2006); L. Gianessi and P. Musumeci, New J. Phys. 8, 294 (2006).

[3] W. D. Kimura, M. Babzien, I. Ben-Zvi, L. P. Campbell, D. B. Cline, C. E. Dilley, J. C. Gallardo, S. C. Gottschalk, K. P. Kusche, R.H. Pantell, I. V. Pogorelsky, D.C. Quimby, J. Skaritka, L. C. Steinhauer, V. Yakimenko, and F. Zhou, Phys. Rev. Lett. 92, 054801 (2004).

[4] Y. Liu, X. J. Wang, D. B. Cline, M. Babzien, J. M. Fang, J. Gallardo, K. Kusche, I. Pogorelsky, J. Skaritka, and A. van Steenbergen, Phys. Rev. Lett. 80, 4418 (1998).

[5] R. W. Schoenlein, S. Chattopadhay, H. H. W. Chong, T. E. Glover, P. A. Heimann, C. V. Shank, A. A. Zholents, and M. S. Zolotarev, Science 287, 2237 (2000).

[6] W. B. Colson, IEEE J. Quantum Electron. 17, 1417 (1981). 
[7] P. Musumeci, C. Pellegrini, and J. B. Rosenzweig, Phys. Rev. E 72, 016501 (2005).

[8] P. Musumeci, S. Ya. Tochitsky, S. Boucher, C. E. Clayton, A. Doyuran, R. J. England, C. Joshi, C. Pellegrini, J.E. Ralph, J. B. Rosenzweig, C. Sung, S. Tolmachev, G. Travish, A. A. Varfolomeev, A. A. Varfolomeev, Jr., T. Yarovoy, and R. B. Yoder, Phys. Rev. Lett. 94, 154801 (2005).

[9] C.M.S. Sears, E. Colby, B. M. Cowan, R. H. Siemann, J. E. Spenser, R. L. Byer, and T. Plettner, Phys. Rev. Lett. 95, 194801 (2005).

[10] A. H. Lumpkin, R. Dejus, W. J. Berg, M. Borland, Y.C. Chae, E. Moog, N. S. Sereno, and B. X. Yang, Phys. Rev. Lett. 86, 79 (2001).

[11] A. Tremaine, X. J. Wang, M. Babzien, I. Ben-Zvi, M. Cornaccia, A. Muroh, H.-D. Nuhn, R. Malone, C. Pellegrini, S. Reiche, J. Rosenzweig, J. Skaritka, and V.
Yakimenko, Phys. Rev. E 66, 036503 (2002).

[12] S. G. Anderson, M. Loh, P. Musumeci, J. B. Rosenzweig, H. Suk, and M. C. Thompson, in Advanced Accelerator Concepts-2000, edited by P.L. Colestock and S. Kelly, AIP Conf. Proc. No. 569 (AIP, New York, 2000), p. 487.

[13] S. Ya. Tochitsky, R. Narang, C. V. Filip, P. Musumeci, C.E. Clayton, R. B. Yoder, K.A. Marsh, J.B. Rosenzweig, C. Pellegrini, and C. Joshi, Phys. Plasmas 11, 2875 (2004).

[14] P. Musimeci, S. Ya. Tochitsky, R. Tikhoplav, J. B. Rosenzweig, and C. Joshi, in Proceedings of the 29th International Free Electron Laser Conference FEL07, Novosibirsk, Russia, 2007, paper WEPPH041, http:// accelconf.web.cern.ch/AccelConf/f07/INDEX.HTM.

[15] L. Giannessi and M. Quattromini, Nucl. Instrum. Methods Phys. Res., Sect. A 436, 443 (1999). 